\title{
Measures of noncompactness in modular spaces and fixed point theorems for multi- valued nonexpansive mappings
}

\author{
T. Domínguez Benavides and P. Lorenzo Ramírez@
}

\begin{abstract}
This paper is devoted to state some fixed point results for multivalued mappings in modular vector spaces. For this purpose, we study the uniform noncompact convexity, a geometric property for modular spaces which is similar to nearly uniform convexity in the Banach spaces setting. Using this property, we state several new fixed point theorems for multivalued nonexpansive mappings in modular spaces.
\end{abstract}

Mathematics Subject Classification. Primary 47H10, 47H04, Secondary $46 \mathrm{E} 30$.

Keywords. Fixed point, multivalued nonexpansive mapping, modular vector space.

\section{Introduction}

The beginning of the Fixed Point Theory for nonexpansive mappings occurred in 1965 when Browder [5], Browder and Göhde [6,18] and Kirk [24] proved that every nonexpansive mapping defined on a convex closed bounded subset of, respectively, a Hilbert space, a uniformly convex Banach space or a reflexive Banach space with normal structure, has a fixed point. A natural problem is to extend these results to multivalued nonexpansive mappings (see Problem 8 in [32]). Using the uniqueness of the asymptotic center of a bounded sequence in a uniformly convex space, Browder-Göhde's Theorem was extended by Lim [27] (see also in [33] Corollary 3.5). Surprisingly, 55 years later, it is still an open problem the possibility of extending Kirk's Theorem. However, some partial extensions have been obtained assuming that the Banach space satisfies several different conditions which imply normal structure (see [9] and references therein). Another direction to research has been the development of the theory for single-valued nonexpansive mappings in modular

The authors are supported by MICIU, Grant PGC2018-098474-B-C2-1 and Andalusian Regional Government Grant FQM-127. 
function spaces (see, for instance [21,22]). Modular spaces were introduced by Nakano [29,30] and developed by Orlicz and Musielak [28]. A very relevant class of modular spaces are the variable exponent Lebesgue spaces, due to their applications to partial differential equations and variational integrals with non-standard growth conditions. This fact, especially after M. Rúžička [34] discovered that they constitute a natural functional setting for the mathematical model of electrorheological fluids, has led to renewed attention on the modular function spaces.

Nearly uniform convexity is a geometric condition that has proved to be successful to obtain fixed points for multivalued nonexpansive mappings in Banach spaces $[12,13]$. Since this condition has a counterpart in modular spaces [21, Section 4.3], it is very natural to study the validity of these results in the setting of modular spaces. After several sections with preliminaries and technical results, in Sect. 6, we state some fixed point results for multivalued nonexpansive mappings in modular spaces. Our approach follows, in some parts, similar arguments to those in $[12,13]$, but, in some other parts, we need some very different techniques. We avoid details in the first case and we will give complete proofs in the latter. We have included two examples of Orlicz sequential variable exponent spaces [31] where our results can be applied.

\section{Preliminaries}

We start by recalling some notions and facts concerning modular spaces. For more details, the reader is referred to [21,23,28-30].

Definition 2.1. Let $\mathcal{X}$ be an arbitrary vector space.

(a) A functional $\rho: \mathcal{X} \rightarrow[0, \infty]$ is called a convex modular if for arbitrary $x, y \in \mathcal{X}$

(i) $\rho(x)=0$ if and only if $x=0$;

(ii) $\rho(\alpha x)=\rho(x)$ for every scalar $\alpha$ with $|\alpha|=1$;

(iii) $\rho(\alpha x+\beta y) \leq \alpha \rho(x)+\beta \rho(y)$ if $\alpha+\beta=1$ and $\alpha, \beta \geq 0$.

(b) A modular $\rho$ defines a corresponding modular space, i.e. the vector space $\mathcal{X}_{\rho}$ given by

$$
\mathcal{X}_{\rho}=\{x \in \mathcal{X}: \rho(x / \lambda)<\infty \text { for some } \lambda>0\} .
$$

Notice that the convexity of the modular implies that the $\rho$-ball $B_{\rho}(x, r)=$ $\left\{y \in \mathcal{X}_{\rho}: \rho(y-x) \leq r\right\}$ is convex for any $x \in \mathcal{X}_{\rho}$ and $r>0$.

The formula

$$
\|x\|_{\rho}=\inf \left\{\alpha>0: \rho\left(\frac{x}{\alpha}\right) \leq 1\right\}
$$

defines a norm which is frequently called the Luxemburg norm. Thus, any convex modular space can be simultaneously studied as a normed space with the Luxemburg norm and any topic on these spaces can be split in two parts corresponding either to the modular space or the normed space. It should be noticed that in non-trivial cases, the Luxemburg norm is particularly difficult 
to compute. Consequently, the results for the norm should be usually deduced from the properties of the modular space.

Definition 2.2. (a) The sequence $\left\{x_{n}\right\}$ in $\mathcal{X}_{\rho}$ is said to be $\rho$-convergent to $x \in \mathcal{X}_{\rho}$ if $\rho\left(x_{n}-x\right) \rightarrow 0$ as $n \rightarrow \infty$.

(b) The sequence $\left\{x_{n}\right\}$ in $\mathcal{X}_{\rho}$ is said to be $\rho$-Cauchy if $\rho\left(x_{n}-x_{m}\right) \rightarrow 0$ as $n$ and $m$ go to $\infty$.

(c) A subset $C$ of $\mathcal{X}_{\rho}$ is called $\rho$-closed if the $\rho$-limit of a $\rho$-convergent sequence of $C$ always belongs to $C$.

(d) $\mathcal{X}_{\rho}$ is said to be $\rho$-complete if every $\rho$-Cauchy sequence is $\rho$-convergent.

(e) A subset $C$ of $\mathcal{X}_{\rho}$ is $\rho$-bounded if

$$
\operatorname{diam}_{\rho}(C)=\sup \{\rho(x-y) ; x, y \in C\}<\infty .
$$

It is clear that the diameter of a set is preserved by translation, i.e., $\operatorname{diam}_{\rho}(C)=\operatorname{diam}_{\rho}(C+x)$ for every $x \in \mathcal{X}_{\rho}$. Therefore, $C$ is $\rho$-bounded if and only if $C+x$ is.

(f) A subset $C$ of $\mathcal{X}_{\rho}$ is called $\rho$-compact if for any $\left\{x_{n}\right\}$ in $C$ there exists a subsequence $\left\{x_{n_{k}}\right\}$ of $\left\{x_{n}\right\}$ and $x \in C$ such that $\lim _{k} \rho\left(x_{n_{k}}-x\right)=0$.

(g) $\rho$ is said to satisfy the Fatou property if for any sequence $\left\{x_{n}\right\}$ in $\mathcal{X}_{\rho}$ $\rho$-convergent to $x \in \mathcal{X}_{\rho}$, we have $\rho(x) \leq \liminf _{n} \rho\left(x_{n}\right)$.

Given a subset $A$ of $\mathcal{X}_{\rho}$, we denote $c o(A)$ its convex hull, i.e., the smallest convex subset of $\mathcal{X}_{\rho}$ containing $A$. From the convexity of the modular $\rho$, we easily deduce that $\operatorname{diam}_{\rho}(c o(A))=\operatorname{diam}_{\rho}(A)$.

Let us describe the relationship between the modular-convergence and norm-convergence in modular spaces. We can use the following proposition:

Proposition 2.3. (Proposition 3.7 and 3.9 in [21]) Let $\rho$ be a convex modular and let $x \in \mathcal{X}_{\rho}$. The following assertions are true:

(a) If $\|x\|_{\rho}<1$ then $\rho(x) \leq\|x\|_{\rho}$.

(b) If $\|x\|_{\rho}>1$ then $\rho(x) \geq\|x\|_{\rho}$.

Remark 2.4. (1) From part (a) of Proposition 2.3 we conclude that a normconvergent sequence in $\mathcal{X}_{\rho}$ is $\rho$-convergent. Therefore, every $\rho$-closed set in $\mathcal{X}_{\rho}$ is norm-closed.

(2) An easy consequence of part (b) of Proposition 2.3 is that any $\rho$-bounded subset of $\mathcal{X}_{\rho}$ is also norm-bounded.

(3) It is easy to check that the Fatou property implies that the $\rho$-balls are $\rho$-closed and that the $\rho$-diameter of a set is the same as the $\rho$-diameter of its $\rho$-closure. The Fatou property also implies that: if $x \in \mathcal{X}_{\rho}$ and $K$ is a nonempty $\rho$-compact subset of $\mathcal{X}_{\rho}$, then there exists $y_{0} \in K$ such that

$$
\rho\left(x-y_{0}\right)=d_{\rho}(x, K),
$$

where $d_{\rho}(x, K)=\inf \{\rho(x, y): y \in K\}$.

In the following, we always assume that the modular is $\rho$-complete. 
Definition 2.5. Let $\rho$ be a convex modular. We say that the modular satisfies the $\Delta_{2}$-type condition if there exists $K>0$ such that $\rho(2 x) \leq K \rho(x)$ for any $x \in \mathcal{X}_{\rho}$.

Definition 2.6. The growth function $\omega_{\rho}:[0, \infty) \rightarrow[0, \infty)$ of a modular $\rho$ is defined as follows:

$$
\omega_{\rho}(t):=\sup \left\{\frac{\rho(t x)}{\rho(x)}: 0<\rho(x)<\infty\right\} \text { for all } t \geq 0 .
$$

Lemma 2.7. [11] Let $\rho$ be a convex modular satisfying the $\Delta_{2}$-type condition. Then the growth function $\omega_{\rho}$ has the following properties:

1. $\omega_{\rho}(t)<\infty$ for every $t \in[0, \infty)$.

2. $\omega_{\rho}(t)=0$ if and only if $t=0$.

3. $\omega_{\rho}:[0, \infty) \rightarrow[0, \infty)$ is a convex, strictly increasing function. So, it is continuous.

4. $\omega_{\rho}(\alpha \beta) \leq \omega_{\rho}(\alpha) \omega_{\rho}(\beta)$ for all $\alpha, \beta \geq 0$.

5. $\omega_{\rho}^{-1}(\alpha) \omega_{\rho}^{-1}(\beta) \leq \omega_{\rho}^{-1}(\alpha \beta)$ for all $\alpha, \beta \geq 0$, where $\omega_{\rho}^{-1}$ is the function inverse of $\omega$.

6. $\|x\|_{\rho} \leq \frac{1}{\omega_{\rho}^{-1}(1 / \rho(x))}$ for every $x \in \mathcal{X}_{\rho} \backslash 0$.

Remark 2.8. It follows from the above lemma that $\rho(x) \leq \omega_{\rho}\left(\|x\|_{\rho}\right)$ for every $x \in \mathcal{X}_{\rho}$. Indeed, for every $\alpha>\|x\|_{\rho}$

$$
\rho(x)=\rho(\alpha x / \alpha) \leq \omega_{\rho}(\alpha) \rho(x / \alpha) \leq \omega_{\rho}(\alpha) .
$$

Letting $\alpha$ go to $\|x\|_{\rho}$ and using the continuity of $\omega_{\rho}(\cdot)$, we obtain the wanted inequality. As a consequence any norm-bounded subset of $\mathcal{X}_{\rho}$ is also $\rho$-bounded.

Furthermore, we also obtain that $\rho$-convergence is identical to normconvergence. Moreover, the modular is $\rho$-complete if and only if it is normcomplete and $\rho$-compact ( $\rho$-closed) sets are the same as norm-compact (normclosed) sets. We will remove the prefix $\rho$ in this case.

The following is a technical lemma which will be needed because of the lack of the triangular inequality.

Lemma 2.9. [11] Assume that $\rho$ is a convex modular satisfying the $\Delta_{2}$-type condition. Let $\left\{x_{n}\right\},\left\{y_{n}\right\}$ two sequences in $\mathcal{X}_{\rho}$. Then

$$
\lim _{n} \rho\left(y_{n}\right)=0 \Longrightarrow \limsup _{n} \rho\left(x_{n}+y_{n}\right)=\limsup _{n} \rho\left(x_{n}\right)
$$

and

$$
\lim _{n} \rho\left(y_{n}\right)=0 \Longrightarrow \liminf _{n} \rho\left(x_{n}+y_{n}\right)=\liminf _{n} \rho\left(x_{n}\right) .
$$

As a result of the above lemma, the modular $\rho$ satisfies the Fatou property under the $\Delta_{2}$-type condition. In fact, $\rho(x)=\lim _{n} \rho\left(x_{n}\right)$ if $\lim _{n} \rho\left(x_{n}-x\right)=0$ and the modular is continuous.

In the remainder of the paper, we will assume that $\mathcal{X}_{\rho}$ satisfies the Fatou property even though no $\Delta_{2}$-type condition is assumed.

Some existence fixed point theorems for nonlinear mappings defined in modular spaces require a kind of uniform continuity of the modular. 
Definition 2.10. A modular $\rho$ is said to be uniformly continuous on bounded sets if for every bounded subset $M$ of $\mathcal{X}_{\rho}$ and for every $\varepsilon>0$, there exists $\delta>0$ such that

$$
|\rho(x+y)-\rho(x)|<\varepsilon
$$

whenever $x \in M, y \in \mathcal{X}_{\rho}, \rho(y)<\delta$.

The following result is a particular case of Lemma 3.4 in [14] (see also $[20])$.

Lemma 2.11. Assume that $\rho$ is a convex modular satisfying the $\Delta_{2}$-type condition. Then

1. $\rho$ is uniformly continuous on bounded sets.

2. For any bounded sequence $\left\{x_{n}\right\} \subset \mathcal{X}_{\rho}$ and any bounded subset $M$ of $\mathcal{X}_{\rho}$, the function $\phi: M \rightarrow \mathbb{R}$ defined by $\phi(x)=\limsup \rho\left(x_{n}-x\right)$ is $\rho$ continuous, i.e., $\phi(y)=\lim _{m} \phi\left(y_{m}\right)$ provided $y, y_{m} \in M$ and $\lim _{m} \rho\left(y_{m}-\right.$ $y)=0$.

The following property can be understood as the modular equivalence of the Banach space reflexivity. It will be a powerful tool to prove the fixed point property in modular spaces.

Definition 2.12. [21] A modular space $\mathcal{X}_{\rho}$ is said to satisfy property $(R)$ if every nonincreasing sequence $\left\{C_{n}\right\}$ of nonempty, $\rho$-bounded, $\rho$-closed, convex subsets of $\mathcal{X}_{\rho}$ has a nonempty intersection.

The method of asymptotic centers has played an important role in the fixed point theory for nonexpansive multivalued mappings in Banach spaces. Some definitions and results concerning asymptotic centers can be adapted to modular spaces in a straightforward way:

Let $C$ be a nonempty $\rho$-closed $\rho$-bounded subset of the space $\mathcal{X}_{\rho}$ and $\left\{x_{n}\right\}$ be a bounded sequence in $\mathcal{X}_{\rho}$. We define

$$
\begin{gathered}
r_{\rho}\left(C,\left\{x_{n}\right\}\right)=\inf \left\{\limsup _{n} \rho\left(x_{n}-x\right): x \in C\right\}, \\
A_{\rho}\left(C,\left\{x_{n}\right\}\right)=\left\{x \in C: \limsup _{n} \rho\left(x_{n}-x\right)=r_{\rho}\left(C,\left\{x_{n}\right\}\right)\right\} .
\end{gathered}
$$

The number $r_{\rho}\left(C,\left\{x_{n}\right\}\right)$ and the (possible empty) set $A_{\rho}\left(C,\left\{x_{n}\right\}\right)$ are called, respectively, the $\rho$-asymptotic radius and the $\rho$-asymptotic center of $\left\{x_{n}\right\}$ in $C$.

Obviously, $A_{\rho}\left(C,\left\{x_{n}\right\}\right)$ is a convex set as $C$ is. Furthermore, the set $A_{\rho}\left(C,\left\{x_{n}\right\}\right)$ is nonempty and closed whenever the space satisfies the $\Delta_{2}$ type condition and satisfies property $(R)$. Indeed, for any $m \geq 1$ consider the set

$$
A_{m}=\left\{y \in C: \limsup _{n} \rho\left(x_{n}-y\right) \leq r+\frac{1}{m}\right\},
$$


where $r=r_{\rho}\left(C,\left\{x_{n}\right\}\right)$. Clearly $A_{m}$ is nonempty and convex. Also, $A_{m}$ is closed by Lemma 2.11. It follows from property $(R)$ that $A_{\rho}\left(C,\left\{x_{n}\right\}\right)=$ $\bigcap A_{m} \neq \emptyset$.

$m \geq 1$

The sequence $\left\{x_{n}\right\}$ is said to be regular relative to $C$ if the asymptotic radii of all subsequences of $\left\{x_{n}\right\}$ (relative to $C$ ) are the same. If, in addition, $A_{\rho}\left(C,\left\{y_{n}\right\}\right)=A_{\rho}\left(C,\left\{x_{n}\right\}\right)$ for every subsequence $\left\{y_{n}\right\}$ of $\left\{x_{n}\right\}$ we say that $\left\{x_{n}\right\}$ is asymptotically uniform relative to $C$.

Similarly as Goebel, Lim and Kirk [15,24,27] did for Banach spaces, the following lemma can be proved:

Lemma 2.13. Let $\rho$ be a convex modular satisfying the $\Delta_{2}$-type condition. Let $C$ be a nonempty closed bounded separable subset of the space $\mathcal{X}_{\rho}$ and $\left\{x_{n}\right\}$ be a bounded sequence in $\mathcal{X}_{\rho}$. Then $\left\{x_{n}\right\}$ contains a regular and asymptotically uniform subsequence relative to $C$.

If $D$ is a $\rho$-bounded subset of $\mathcal{X}_{\rho}$, the $\rho$-Chebyshev radius of $D$ relative to $C$ is defined by

$$
r_{\rho}(C, D):=\inf \{\sup \{\rho(x-y): y \in D\}: x \in C\} .
$$

Notice that $r_{\rho}(C, D) \leq \operatorname{diam}_{\rho}(D)$ if $D \subset C$. Moreover, if $\mathcal{X}_{\rho}$ satisfies the $\Delta_{2}$-type condition then $\operatorname{diam}_{\rho}(D) \leq \omega(2) r_{\rho}(C, D)$.

Let $C$ be a nonempty subset of the space $\mathcal{X}_{\rho}$. We shall denote by $F_{\rho}(C)$ the family of all nonempty $\rho$-closed subsets of $C$ and by $K_{\rho}(C)$ (resp. $\left.K C_{\rho}(C)\right)$ the family of all nonempty $\rho$-compact subsets of $C$ (resp. $\rho$-compact convex). We can define the analogue to the Hausdorff distance for modular spaces by

$$
H_{\rho}(A, B):=\max \left\{\sup _{a \in A} d_{\rho}(a, B), \sup _{b \in B} d_{\rho}(b, A)\right\}, \quad A, B \in F_{\rho}(C),
$$

where for $x \in \mathcal{X}_{\rho}$ and $E \subset \mathcal{X}_{\rho} d_{\rho}(x, E):=\inf \{\rho(x-y): y \in E\}$ is the $\rho$-distance from the point $x$ to the subset $E$. This function will be called Hausdorff $\rho$-distance even though it is not a metric.

If $C$ is a $\rho$-closed convex subset of $\mathcal{X}_{\rho}$, then a multivalued mapping $T: C \rightarrow F_{\rho}\left(\mathcal{X}_{\rho}\right)$ is said to be $\rho$-contractive if there exists a constant $k \in[0,1)$ such that

$$
H_{\rho}(T x, T y) \leq k \rho(x-y), \quad x, y \in C,
$$

and $T$ is said to be $\rho$-nonexpansive if

$$
H_{\rho}(T x, T y) \leq \rho(x-y), \quad x, y \in C .
$$

Finally, we say that $x \in C$ is a fixed point of $T$ if and only if $x \in T x$. A counterpart of the Banach Contraction Principle for multivalued mappings in modular spaces has been proved in [7].

Theorem 2.14. Let $\rho$ be a convex modular satisfying the $\Delta_{2}$-type condition, $C$ a nonempty bounded closed subset of $\mathcal{X}_{\rho}$, and $T: C \rightarrow F_{\rho}(C)$ a $\rho$-contraction mapping. Then $T$ has a fixed point. 


\section{Measures of noncompactness in modular spaces}

The definitions of both Kuratowski and Hausdorff measure of noncompactness were introduced in modular function spaces by Khamsi and Kozlowski [21]. Similarly, we can extend such concepts to modular abstract spaces in the following way.

Definition 3.1. Let $\rho$ be a convex modular and $\mathcal{B}$ the family of all nonempty $\rho$-bounded subset of $\mathcal{X}_{\rho}$. Define the Kuratowski measure of noncompactness of $A \in \mathcal{B}$ by

$$
\begin{gathered}
\alpha(A)=\inf \{\varepsilon>0: A \text { can be covered by a finite number of sets } \\
\text { with } \rho-\text { diameter smaller than } \varepsilon\},
\end{gathered}
$$

and the Hausdorff measure of noncompactness of $A$ by

$$
\begin{gathered}
\chi(A)=\inf \{\varepsilon>0: A \text { can be covered by a finite number of } \\
\rho-\text { balls of radius smaller than } \varepsilon\} .
\end{gathered}
$$

We will make the obvious convention that the infimum over an empty set is infinite.

Note that $\chi(A) \leq \alpha(A) \leq \operatorname{diam}_{\rho}(A)$. If in addition $\rho$ satisfies the $\Delta_{2^{-}}$ type condition, then $\alpha(A) \leq \omega(2) \chi(A)$.

We next summarize the basic properties of the above measures of noncompactness. These properties follow immediately from the definitions and some of them have been proved in [21].

Proposition 3.2. Let $\phi$ denote $\alpha$ or $\chi$. Then the following properties hold:

1 If $A \subset B$ then $\phi(A) \leq \phi(B)$ for all $A, B \in \mathcal{B}$.

$2 \phi\left(\bar{A}^{\rho}\right)=\phi(A)$ for all $A \in \mathcal{B}$, where $\bar{A}^{\rho}$ denotes the $\rho$-closure of $A$.

$3 \phi(A \cup B)=\max \{\phi(A), \phi(B)\}$ for all $A, B \in \mathcal{B}$.

4 If $A$ is a finite set, then $\phi(A)=0$.

$5 \phi(A+x)=\phi(A)$ for any $x \in \mathcal{X}_{\rho}$.

$6 \phi(-A)=\phi(A)$.

$7 \phi(\lambda A+\mu B) \leq \lambda \phi(A)+\mu \phi(B)$ if $\lambda+\mu=1$ and $\lambda, \mu \geq 0$ for all $A, B \in \mathcal{B}$.

8 If $\alpha(A)=0$, then $\bar{A}^{\rho}$ is $\rho$-compact. If $\rho$ is a convex modular satisfying the $\Delta_{2}$-type condition, $\chi(A)=0$ implies $\bar{A}^{\rho}$ is $\rho$-compact. Conversely, if $\rho$ is a convex modular satisfying the $\Delta_{2}$-type condition and $A$ is $\rho$ compact, then $\phi(A)=0$.

9 If $\left\{A_{n}\right\}$ is a decreasing sequence of nonempty $\rho$-closed, $\rho$-bounded subset of $\mathcal{X}_{\rho}$ and $\lim _{n} \phi\left(A_{n}\right)=0$, then $A_{\infty}:=\cap_{n \geq 1} A_{n}$ is nonempty. If $\phi=\alpha$, then $A_{\infty}$ is $\rho$-compact. If, in addition, $\rho$ satisfies the $\Delta_{2}$-type condition, and $\phi=\chi$ then $A_{\infty}$ is $\rho$-compact.

Let $\rho$ a modular satisfying the $\Delta_{2}$-type condition. Assume that $C$ is a subset of the modular space $\mathcal{X}_{\rho}$. We can consider the Hausdorff measure of noncompactness $\chi_{C}$ defined for any $\rho$-bounded subset $A$ of $C$ by $\chi_{C}(A)=$ $\inf \{\varepsilon>0: A$ can be covered by finitely many $\rho$-balls centered at points in $C$ with radius less than $\varepsilon$. It must be noted that this measure depends on $C$ and it is, in general, different from $\chi=: \chi_{\mathcal{X}}$. However, if $C$ is a convex 
closed set, it is easy to check that the arguments to prove the properties in Proposition 3.2 equally well apply to the measure $\chi_{C}$.

We are going to prove that the Kuratowski and Hausdorff measure of noncompactness are invariant under passage to the convex hull.

Theorem 3.3. Let $\rho$ be a convex modular satisfying the $\Delta_{2}$-type condition. Let $\phi$ denote $\alpha$ or $\chi$, then $\phi(\operatorname{co}(A))=\phi(A)$ for all $A \in \mathcal{B}$.

Proof. We prove the result for $\chi$. The proof for $\alpha$ is analogous. Since $A \subset$ $\operatorname{co}(A)$ we obtain $\chi(A) \leq \chi(\operatorname{co}(A))$. If $\chi(A)<\chi(\operatorname{co}(A))$, there exists $\lambda>1$ such that $\chi(\operatorname{co}(A))>\lambda \chi(A)$. Choose $\alpha \in(0,1)$ such that $\alpha \omega\left(\frac{1}{\alpha}\right) \leq \lambda$. We are going to follow an argument as in [2] to get a contradiction. Indeed, for every $\varepsilon>0$ there exist $x_{1}, \cdots, x_{n} \in \mathcal{X}_{\rho}$ such that

$$
A \subset \bigcup_{i=1}^{n} B_{\rho}\left(x_{i}, \chi(A)+\varepsilon\right) \text {. }
$$

Let us define

$$
\Delta=\left\{\left(\sigma_{1}, \cdots, \sigma_{n}\right): \sum_{i=1}^{n} \sigma_{i}=1, \sigma_{i} \geq 0 \forall i=1 \cdots, n\right\}
$$

and $\Delta(\sigma)=\sum_{i=1}^{n} \sigma_{i} B_{\rho}\left(x_{i}, \chi(A)+\varepsilon\right)$. As proved in [2, Theorem 2.4], the set $\bigcup_{\sigma \in \Delta} \Delta(\sigma)$ is convex.

$$
\begin{gathered}
\text { Since } A \subset \bigcup_{i=1}^{n} B_{\rho}\left(x_{i}, \chi(A)+\varepsilon\right) \subset \bigcup_{\sigma \in \Delta} \Delta(\sigma) \text { it follows that } \\
\operatorname{co}(A) \subset \bigcup_{\sigma \in \Delta} \Delta(\sigma) .
\end{gathered}
$$

Since $\Delta$ is compact, we can find $\sigma^{(1)}, \cdots, \sigma^{(m)} \in \Delta$ such that for all $\sigma \in \Delta$, we have

$$
\min \left\{\left\|\sigma-\sigma^{(j)}\right\|_{1}: j=1, \cdots, m\right\}<\min \left\{\frac{\varepsilon}{M}, 1\right\}=\varepsilon^{\prime},
$$

where $M=\sup \left\{\rho(x): x \in B_{\rho}\left(x_{i}, \chi(A)+\varepsilon\right), i=1 \cdots, n\right\}<+\infty$.

Let $x$ be any element of $\operatorname{co}(A)$. Since $x=\sum_{i=1}^{n} \sigma_{i} y_{i}$, for some $\sigma=$ $\left(\sigma_{1}, \cdots, \sigma_{n}\right) \in \Delta$ and $y_{i} \in B_{\rho}\left(x_{i}, \chi(A)+\varepsilon\right), i=1, \cdots, n$, there exists $j \in\{1, \cdots, m\}$ such that $\sum_{i=1}^{n}\left|\sigma_{i}-\sigma_{i}^{j}\right|<\varepsilon^{\prime}$.

$$
\begin{aligned}
& \text { Denote } \bar{x}=\sum_{i=1}^{n} \sigma_{i}^{j} y_{i} \in \Delta\left(\sigma^{j}\right) . \text { We have } \\
& \rho(x-\bar{x})=\rho\left(\sum_{i=1}^{n}\left(\sigma_{i}-\sigma_{i}^{j}\right) y_{i}\right)=\rho\left(\sum_{i=1}^{n}\left(\left|\sigma_{i}-\sigma_{i}^{j}\right| \operatorname{sign}\left(\sigma_{i}-\sigma_{i}^{j}\right) y_{i}\right)\right) .
\end{aligned}
$$


Put $z_{i}=\operatorname{sign}\left(\sigma_{i}-\sigma_{i}^{j}\right) y_{i}$. Since $\sum_{i=1}^{n}\left|\sigma_{i}-\sigma_{i}^{j}\right| \leq \varepsilon^{\prime} \leq 1$, we apply the convexity of the modular to obtain

$$
\rho(x-\bar{x}) \leq \sum_{i=1}^{n}\left|\sigma_{i}-\sigma_{i}^{j}\right| \rho\left(z_{i}\right)=\sum_{i=1}^{n}\left|\sigma_{i}-\sigma_{i}^{j}\right| \rho\left(y_{i}\right) .
$$

Hence,

$$
\rho(x-\bar{x}) \leq \sum_{i=1}^{n}\left|\sigma_{i}-\sigma_{i}^{j}\right| M<\varepsilon
$$

Furthermore,

$$
\begin{aligned}
\rho\left(x-\sum_{i=1}^{n} \sigma_{i}^{j} x_{i}\right) & =\rho\left(x-\bar{x}+\bar{x}-\sum_{i=1}^{n} \sigma_{i}^{j} x_{i}\right) \\
& =\rho\left(x-\bar{x}+\sum_{i=1}^{n} \sigma_{i}^{j}\left(y_{i}-x_{i}\right)\right) \\
& \leq \alpha \omega\left(\frac{1}{\alpha}\right) \rho\left(\sum_{i=1}^{n} \sigma_{i}^{j}\left(y_{i}-x_{i}\right)\right) \\
& +(1-\alpha) \omega\left(\frac{1}{1-\alpha}\right) \rho(x-\bar{x}) \\
& \leq \lambda(\chi(A)+\varepsilon)+(1-\alpha) \omega\left(\frac{1}{1-\alpha}\right) \varepsilon
\end{aligned}
$$

Therefore,

$$
\operatorname{co}(A) \subset \bigcup_{j=1}^{m}\left[B_{\rho}\left(\sum_{i=1}^{n} \sigma_{i}^{j} x_{i}, \lambda(\chi(A)+\varepsilon)+(1-\alpha) \omega\left(\frac{1}{1-\alpha}\right) \varepsilon\right)\right] .
$$

This implies that

$$
\begin{aligned}
\chi(c o(A)) & \leq \max _{1 \leq j \leq m} \chi\left(B_{\rho}\left(\sum_{i=1}^{n} \sigma_{i}^{j} x_{i}, \lambda(\chi(A)+\varepsilon)+(1-\alpha) \omega\left(\frac{1}{1-\alpha}\right) \varepsilon\right)\right) \\
& \leq \lambda(\chi(A)+\varepsilon)+(1-\alpha) \omega\left(\frac{1}{1-\alpha}\right) \varepsilon .
\end{aligned}
$$

Bearing in mind that $\varepsilon$ was chosen arbitrary, we obtain $\chi(\operatorname{co}(A) \leq \lambda \chi(A)$ which is a contradiction.

\section{Uniform noncompact convexity in modular spaces}

Similarly as Goebel and Sekowski [17] did for Banach spaces, we can define a scaling of the convexity for modular spaces using the measures of noncompactness. 
Definition 4.1. [21] Let $\rho$ be a convex modular. Let $\phi$ denote $\alpha$ or $\chi$. The $\rho$ modulus of noncompact convexity associated with $\phi$ is defined in the following way:

$$
\begin{aligned}
\Delta_{\phi}(r, \varepsilon)=\sup \{c>0 & \text { : for any } \rho \text {-bounded convex } A \subset \mathcal{X}_{\rho} \text { and } x \in \mathcal{X}_{\rho} \\
& \text { such that } A \subset B_{\rho}(x, r) \text { with } \phi(A) \geq r \varepsilon, \\
& \text { then } \left.d_{\rho}(x, A) \leq(1-c) r\right\},
\end{aligned}
$$

for every $r>0, \varepsilon>0$.

Remark 4.2. Since both measures of noncompactness $\alpha$ and $\chi$ are invariants under translations and $d_{\rho}(0, A)=d_{\rho}(x, A+x)$ for every set $A \subset \mathcal{X}_{\rho}$ and every $x \in \mathcal{X}_{\rho}$, Definition 4.1 is equivalent to the following:

Definition 4.3. Let $\rho$ be a convex modular. Let $\phi$ denote $\alpha$ or $\chi$. The $\rho$ modulus of noncompact convexity associated with $\phi$ is defined in the following way:

$$
\begin{gathered}
\Delta_{\phi}(r, \varepsilon)=\sup \left\{c>0 \text { : for any } \rho \text {-bounded convex } A \subset B_{\rho}(0, r)\right. \text { with } \\
\left.\phi(A) \geq r \varepsilon, \text { then } d_{\rho}(0, A) \leq(1-c) r\right\},
\end{gathered}
$$

for every $r>0, \varepsilon>0$.

Observe that the function $\Delta_{\chi}(r, \cdot)$ is defined for $\varepsilon \in(0,1]$. If $\rho$ satisfies the $\Delta_{2}$-type condition, then $\Delta_{\alpha}(r, \cdot)$ is defined for $\varepsilon \in(0, \omega(2)]$. It is clear that both functions are non-decreasing in $\varepsilon$.

The $\rho$-characteristic of noncompact convexity of $\mathcal{X}_{\rho}$ associated with the measure of noncompactness $\phi$ is defined by

$$
\varepsilon_{\phi}(r)=\sup \left\{\varepsilon \geq 0: \Delta_{\phi}(r, \varepsilon)=0\right\},
$$

for every $r>0$.

Definition 4.4. Let $\rho$ be a convex modular. The modular space $\mathcal{X}_{\rho}$ is said to be $\phi$-uniformly $\rho$-noncompact convex ( $\phi$-UNC in short) if $\varepsilon_{\phi}(r)=0$ for every $r>0$.

Remark 4.5. It is easy to check that $\Delta_{\alpha}(r, \varepsilon) \leq \Delta_{\chi}(r, \varepsilon)$. Consequently, $\varepsilon_{\alpha}(r) \geq \varepsilon_{\chi}(r)$. Clearly if $\mathcal{X}_{\rho}$ is $\alpha$-uniformly $\rho$-noncompact convex, then $\mathcal{X}_{\rho}$ is $\chi$-uniformly $\rho$-noncompact convex. Moreover, If $\rho$ satisfies the $\Delta_{2}$-type condition $\Delta_{\chi}\left(r, \frac{\varepsilon}{\omega(2)}\right) \leq \Delta_{\alpha}(r, \varepsilon)$ and both concepts are equivalent.

Remark 4.6. In [1] (see also [5,21]), the authors introduced some interlinked notions of $\rho$-uniform convexity in a modular space. One of them leads to define the $\rho$-modulus of uniform convexity for every $r>0$ and $\varepsilon>0$ as follows:

$$
\delta_{1}(r, \varepsilon)=\inf \left\{1-\frac{1}{r} \rho\left(\frac{x+y}{2}\right)\right\},
$$

where the infimum is taken over all $x, y \in \mathcal{X}_{\rho}$ such that $\rho(x) \leq r, \rho(y) \leq r$ and $\rho(x-y) \geq \varepsilon r$. 
According to [1, Definition 3.1] (see also [21]) a modular $\rho$ satisfies $(U U C 1)$ if for every $s \geq 0$ and $\varepsilon>0$, there exist $\eta(s, \varepsilon)>0$ such that

$$
\delta_{1}(r, \varepsilon)>\eta(s, \varepsilon)>0, \text { for } r>s .
$$

Let us show the connection between this modulus and the $\rho$-modulus of noncompact convexity associated with $\phi=\alpha$ or $\chi$.

Let $A \subset \mathcal{X}_{\rho}$ be nonempty $\rho$-bounded convex such that $A \subset B_{\rho}(0, r)$ with $\phi(A) \geq r \varepsilon, r>0$ and $\varepsilon>0$. For $\zeta<1$, by definition of $\phi$, we can find $y_{1}, y_{2} \in A$ such that $\rho\left(y_{1}-y_{2}\right) \geq \zeta \varepsilon r$. Therefore, we have

$$
\rho\left(\frac{y_{1}+y_{2}}{2}\right) \leq r\left(1-\delta_{1}(r, \zeta \varepsilon)\right) .
$$

Since $\frac{y_{1}+y_{2}}{2} \in A$, we deduce that

$$
d_{\rho}(0, A) \leq r\left(1-\delta_{1}(r, \zeta \varepsilon)\right)
$$

This implies

$$
\left.\delta_{1}(r, \zeta \varepsilon)\right) \leq \Delta_{\alpha}(r, \varepsilon),
$$

for every $r>0, \varepsilon>0$ and $\zeta<1$.

Consequently, the class of $\phi$-uniformly $\rho$-noncompact convex spaces includes all $\rho$ - $(U U C 1)$ spaces. The following example shows that the converse of this assertion is not true.

Example 4.7. A uniformly $\rho$-noncompact convex space which is not $\rho$-uniformly convex.

Let $\left\{p_{n}\right\}$ be a sequence in $[1, \infty)$ such that $1<p=: \liminf _{n} p_{n} \leq$ $\lim \sup _{n} p_{n}<\infty$. Consider the Orlicz space $\ell^{p_{n}}$ for the modular

$$
\rho(x)=\sum_{n=1}^{\infty}|x(n)|^{p_{n}} .
$$

It is well known that $\left(\ell^{p_{n}}, \rho\right)$ is a modular sequence space which satisfies the $\Delta_{2}$-type condition. Furthermore, $\left(\ell^{p_{n}},\|\cdot\|_{\rho}\right)$ is a reflexive Banach space whenever $\|\cdot\|_{\rho}$ is the corresponding Luxemburg norm [10, Theorem 18]. Note that $\left(\ell^{p_{n}}, \rho\right)$ does not satisfy any uniform convexity condition (see [1, Definition 3.1]) whenever the sequence $\left\{p_{n}\right\}$ attains the value 1 more than once, because it contains $\mathbb{R}^{2}$ with the 1 -norm. It is clear that the modular is additive for disjointedly supported vectors. On the other hand, the functional $\Lambda_{k}: \ell^{p_{n}} \rightarrow \mathbb{R}$ defined by $\Lambda_{k}(x)=x(k)$ is continuous and so every weakly null sequence in $\left(\ell^{p_{n}},\|\cdot\|_{\rho}\right)$ converges to 0 coordinate-wise.

We are going to prove that $\Delta_{\chi}(\varepsilon, r) \geq \varepsilon / 2$. To do that, let $A$ be a convex subset of the closed ball $B_{\rho}(0, r)$ such that $\chi(A)>\varepsilon r$. We can find a sequence $\left\{x_{n}\right\}$ in $A$ such that $\operatorname{sep}_{\rho}\left(x_{n}\right)=\inf \left\{\rho\left(x_{n}-x_{m}\right): n, m \in \mathbb{N}\right\} \geq \varepsilon r$. Taking a subsequence, we can assume that $\left\{x_{n}\right\}$ is weakly convergent, say to $x \in \bar{A}$, and $\lim _{n} \rho\left(x_{n}-x\right)=: l r$ does exist. After a further subsequence, we can assume that there exist two sequences $\left\{u_{n}\right\},\left\{v_{n}\right\}$ such that $\lim _{n} \rho\left(x-u_{n}\right)=0$, $\lim _{n} \rho\left(x_{n}-x-v_{n}\right)=0, \operatorname{supp} u_{n}<\operatorname{supp} v_{n}$ and supp $v_{n}<\operatorname{supp} v_{n+1}$ for every $n \in \mathbb{N}$, where $\operatorname{supp}(x):=\{n \in \mathbb{N}: x(n) \neq 0\}$ and supp $u<$ 
supp $v$ means that there exists $N \in \mathbb{N}$ such that supp $u \subset[1, N]$ and supp $v \subset[N+1,+\infty)$. Choose an arbitrary $\eta>0$. Using Lemma 2.11, we can choose $n, m$ large enough such that

$$
\begin{gathered}
\varepsilon r-\eta<\rho\left(v_{n}-v_{m}\right) \\
\left|\rho\left(v_{n}\right)-l r\right|<\eta \\
\left|\rho\left(u_{n}\right)-\rho(x)\right|<\eta \\
\rho\left(x_{n}\right) \geq \rho\left(v_{n}+u_{n}\right)-\eta .
\end{gathered}
$$

Thus,

$$
\varepsilon r-\eta<\rho\left(v_{n}-v_{m}\right)=\rho\left(v_{n}\right)+\rho\left(v_{m}\right) \leq 2 l r+2 \eta .
$$

Moreover,

$$
r \geq \rho\left(x_{n}\right) \geq \rho\left(v_{n}+u_{n}\right)-\eta=\rho\left(v_{n}\right)+\rho\left(u_{n}\right)-\eta \geq l r+\rho(x)-3 \eta \geq \frac{\varepsilon}{2} r+\rho(x)-6 \eta .
$$

Hence, $\rho(x) \leq r(1-\varepsilon / 2)+6 \eta$. Since $\eta$ is arbitrary, we have $d_{\rho}(0, A) \leq$ $r(1-\varepsilon / 2)$ which implies $\Delta_{\chi}(\varepsilon, r) \geq \varepsilon / 2$.

Next lemma provides an important tool to prove the relationship between modular uniform noncompact convexity and property $(R)$ as well as one of the main fixed point theorems in our paper (Theorem 6.7 below).

Lemma 4.8. Let $\rho$ be a convex modular satisfying the $\Delta_{2}$-type condition. Assume that $\mathcal{X}_{\rho}$ is $\phi-U N C$. Let $s \in(0,+\infty)$ and $\varepsilon>0$. Then there exists $\lambda<1$ and $\eta>0$ such that, for every $r \in(s, s+\eta)$, we have $1-\Delta_{\phi}(r, \varepsilon) \leq \lambda$.

Proof. Choose $\lambda \in\left(1-\Delta_{\phi}\left(s, \frac{\varepsilon}{\omega(2)}\right), 1\right)$. Since $\frac{\omega(t)}{t} \rightarrow 1$ as $t \rightarrow 1$, we can find $\eta \in(0, s)$ such that

$$
\omega\left(\frac{r}{s}\right) \frac{s}{r}<\frac{\lambda}{1-\Delta_{\phi}\left(s, \frac{\varepsilon}{\omega(2)}\right)}
$$

if $r \in(s, s+\eta)$. Assume that $A \subset B_{\rho}(0, r)$ is a convex set such that $\phi(A) \geq r \varepsilon$. Consider the set $B=\frac{s}{r} A$. We have that $B \subset B_{\rho}(0, s)$ and $\phi(B) \geq \frac{r \varepsilon}{\omega\left(\frac{r}{s}\right)} \geq \frac{r \varepsilon}{\omega(2)}$. Thus,

$$
d_{\rho}(0, B) \leq s\left(1-\Delta_{\phi}\left(s, \frac{\varepsilon}{\omega(2)}\right)\right)
$$

which implies

$$
d_{\rho}(0, A) \leq s \omega\left(\frac{r}{s}\right)\left(1-\Delta_{\phi}\left(s, \frac{\varepsilon}{\omega(2)}\right)\right)<\lambda r .
$$

Therefore, $1-\Delta_{\phi}(r, \varepsilon) \leq \lambda$. 
Theorem 4.9. Let $\rho$ be a convex modular satisfying the $\Delta_{2}$-type condition. Assume that $\mathcal{X}_{\rho}$ is $\phi$-UNC. Then, for any closed bounded convex subset of $\mathcal{X}_{\rho}$ and $x \in \mathcal{X}_{\rho}$, the set

$$
P_{\rho}(x, C)=\left\{y \in C: d_{\rho}(x, C)=\rho(x-y)\right\}
$$

is nonempty compact and convex.

Proof. Since $C$ is closed, we may assume without loss of generality that $d:=$ $d_{\rho}(x, C)>0$. Consider the sets $C_{n}=C \cap B_{\rho}\left(x, d+\frac{1}{n}\right)$ for any $n \geq 1$. Clearly $\left\{C_{n}\right\}$ is a decreasing sequence of closed bounded convex subsets of $\mathcal{X}_{\rho}$ and $P_{\rho}(x, C)=\bigcap_{n \geq 1} C_{n}$.

Assume that $\varepsilon:=\inf _{n} \phi\left(C_{n}\right)=\lim _{n} \phi\left(C_{n}\right)>0$. We have

$$
d_{\rho}\left(x, C_{n}\right) \leq\left(1-\Delta_{\phi}\left(d+\frac{1}{n}, \frac{\varepsilon}{d+1}\right)\right)\left(d+\frac{1}{n}\right) .
$$

According to Lemma 4.8, there exists $\lambda:=\lambda(d, \varepsilon)<1$ and $n_{0}$ such that for every $n \geq n_{0}$

$$
d_{\rho}\left(x, C_{n}\right) \leq \lambda\left(d+\frac{1}{n}\right) .
$$

Since $d \leq d_{\rho}\left(x, C_{n}\right)$, we obtain

$$
d \leq \lambda\left(d+\frac{1}{n}\right)
$$

contradicting the fact that $d>0$. Hence $\lim _{n} \phi\left(C_{n}\right)=0$. By Proposition 3.2 , we deduce that $\bigcap C_{n}$ is a nonempty $\rho$-compact convex subset of $C$ and the proof is complete.

Theorem 4.10. Let $\rho$ be a convex modular satisfying the $\Delta_{2}$-type condition. Assume that $\mathcal{X}_{\rho}$ is $\phi$-UNC. Then $\mathcal{X}_{\rho}$ has the property $(R)$.

Proof. Let $\left\{C_{n}\right\}_{n \geq 1}$ be a decreasing sequence of closed bounded convex subsets of $\mathcal{X}_{\rho}$. Fix $x \in C_{1}$. We have

$$
r=\sup _{n \geq 1} d_{\rho}\left(x, C_{n}\right) \leq \operatorname{diam}_{\rho}\left(C_{1}\right) .
$$

From Theorem 4.9, for every $n \geq 1$ the set $K_{n}=C_{n} \cap B_{\rho}(x, r)$ is nonempty. Clearly, $\left\{K_{n}\right\}$ is a decreasing sequence of closed bounded convex subsets of $\mathcal{X}_{\rho}$. Following the same argument as in the proof of Theorem 4.9, we can show that $\lim _{n} \phi\left(K_{n}\right)=0$. Indeed, if $\varepsilon:=\inf _{n} \phi\left(K_{n}\right)=\lim _{n} \phi\left(K_{n}\right)>0$, we have

$$
d_{\rho}\left(x, K_{n}\right) \leq\left(1-\Delta_{\phi}\left(r, \frac{\varepsilon}{r}\right)\right) r
$$


for all $n \geq 1$. Taking supremum and bearing in mind that $\Delta_{\phi}\left(r, \frac{\varepsilon}{r}\right)>0$ we get a contradiction. So, $\bigcap_{n \geq 1} K_{n}$ is a nonempty $\rho$-compact convex subset of $\mathcal{X}_{\rho}$. Therefore, $\bigcap_{n \geq 1} C_{n}$ is nonempty.

Remark 4.11. In [21], Theorems 4.9 and 4.10 are stated without assuming the $\Delta_{2}$-type condition, but it is not clear for us their validity, because a positive lower bound of $\Delta_{\phi}(r+1 / n, \varepsilon)$, independent of $n$, is needed in both proofs. To obtain this lower bound, we have proved a kind of continuity of the modulus with respect to the first variable from the $\Delta_{2}$-type condition (Lemma 4.8). This bound can be also obtained if we, additionally, assume in the definition of uniform $\rho$-noncompact convexity that there is function $\eta(\cdot, \cdot)$ such that $\Delta_{\phi}(r, \varepsilon)>\eta(s, \varepsilon)>0$ for every $r>s$ and $\varepsilon>0$. (Compare with Theorem 4.1 and Theorem 4.2 in the same monograph when condition (UUC2) is assumed).

\section{Asymptotic centers in UNC spaces}

In this section, we shall give a connection between the asymptotic center of a sequence and the $\rho$-modulus of noncompact convexity. As in the Banach space setting it will play a crucial role to prove the existence of fixed point for multivalued $\rho$-nonexpansive mappings.

First, we state the following lemma.

Lemma 5.1. Let $\rho$ be a convex modular satisfying the $\Delta_{2}$-type condition and $C$ a closed convex subset of $\mathcal{X}_{\rho}$. Let $\left\{x_{n}\right\}$ be a bounded sequence in $C$ and $W$ the convex closed hull of its terms. Define $\Phi: W \rightarrow[0, \infty)$ by $\Phi(x)=$ $\lim \sup _{n} \rho\left(x_{n}-x\right)$. Let $\left\{z_{k}\right\}$ be a sequence in $W$ such that $z_{k} \in A_{k}=: \overline{c o}\left\{x_{n}\right.$ : $n \geq k\}$ (in particular, $z_{k}$ can be equal to $x_{k}$ ) and $z \in \cap_{k=1}^{\infty} \overline{c o}\left\{z_{j}: j \geq k\right\}$. Then

$$
\Phi(z) \leq \limsup _{k} \Phi\left(z_{k}\right)
$$

Proof. Choose $a>\lim \sup _{k} \Phi\left(z_{k}\right)$. For $k$ large enough, $\Phi\left(z_{k}\right)<a$, i.e. $z_{k} \in$ $\Phi^{-1}([0, a])$. Note that $\Phi^{-1}([0, a])$ is a convex set. Since from Lemma 2.11 the function $\Phi$ is continuous, we have that $\Phi^{-1}([0, a])$ is also a closed set which contains $\left\{z_{j}: j>k\right\}$. Thus, it contains the closed set $\overline{c o}\left\{z_{j}: j \geq k\right\}$. In particular, it contains $z$.

Before we present our result, we need the theorem below. Even though this theorem is a direct adaptation of Theorem 5 from [8] for the modular $\rho$, we include its proof for the sake of completeness.

We shall use a simple version of Ramsey Lemma. By $\mathbb{N}$ we denote the set of nonnegative integers, $[\mathbb{N}]$ the collection of its infinite subsets and for 
every set $C$ in $[\mathbb{N}],[C]^{2}$ will denote the set formed by all ordered pair formed with numbers in $C$.

Ramsey's Lemma. Let $f:[\mathbb{N}]^{2} \rightarrow\{1,2\}$ be a function. Then there exists $C \in[\mathbb{N}]$ such that the restriction of $f$ to $[C]^{2}$ is a constant.

Theorem 5.2. Let $\rho$ be a convex modular and $\left\{x_{n}\right\}$ a bounded sequence in $\mathcal{X}_{\rho}$. Then there exists a subsequence $\left\{z_{n}\right\}$ of $\left\{x_{n}\right\}$ such that the limit $\lim _{n \neq m} \rho\left(z_{n}-\right.$ $\left.z_{m}\right)$ exists.

Proof. For every subsequence $\left\{y_{n}\right\}$ of $\left\{x_{n}\right\}$, we denote

$$
\begin{aligned}
\psi\left(\left\{y_{n}\right\}\right)=\inf \{\varepsilon>0: & \left\{y_{n}\right\} \text { can be covered by finitely many sets } \\
& \text { with diameter } \leq \varepsilon\} .
\end{aligned}
$$

Claim. There exists a subsequence $\left\{y_{n}\right\}$ of $\left\{x_{n}\right\}$ such that $\psi\left(\left\{z_{n}\right\}\right)=$ $\psi\left(\left\{y_{n}\right\}\right)$ for every subsequence $\left\{z_{n}\right\}$ of $\left\{y_{n}\right\}$.

To prove the claim, define by induction $\left\{z_{n}^{0}\right\}=\left\{x_{n}\right\}$ and

$$
\psi_{m+1}=\inf \left\{\psi\left(\left\{z_{n}\right\}\right):\left\{z_{n}\right\} \text { is a subsequence of }\left\{z_{n}^{m}\right\}\right\} \text {. }
$$

Let $\left\{z_{n}^{m+1}\right\}$ be a subsequence of $\left\{z_{n}^{m}\right\}$ such that

$$
\psi\left(\left\{z_{n}^{m+1}\right\}\right)<\psi_{m+1}+\frac{1}{m+1} .
$$

Consider the diagonal subsequence $\left\{z_{n}^{n}\right\}$. We will show that this sequence satisfies the required condition. Since $\left\{z_{n}^{n}\right\}$ is a subsequence of $\left\{z_{n}^{m}\right\}$ for $n>m$ we have $\psi\left(\left\{z_{n}^{n}\right\}\right) \leq \psi\left(\left\{z_{n}^{m}\right\}\right)$ for each $m$. Assume that $\left\{z_{n}\right\}$ is a subsequence of $\left\{z_{n}^{n}\right\}$. Hence, $\left\{z_{n}\right\}$ is a subsequence of $\left\{z_{n}^{m}\right\}$ for $n>m$. Thus,

$$
\psi\left(\left\{z_{n}^{n}\right\}\right) \leq \psi\left(\left\{z_{n}^{m}\right\}\right)<\psi_{m}+\frac{1}{m} \leq \psi\left(\left\{z_{n}\right\}\right)+\frac{1}{m} .
$$

Since $m$ is arbitrary, we obtain

$$
\psi\left(\left\{z_{n}^{n}\right\}\right) \leq \psi\left(\left\{z_{n}\right\}\right) \leq \psi\left(\left\{z_{n}^{n}\right\}\right)
$$

and the claim is proved.

Choose now an arbitrary $\varepsilon>0$ and a subsequence $\left\{y_{n}\right\}$ of $\left\{x_{n}\right\}$ satisfying the property in the claim. Taking a subsequence (which "a fortiori" satisfies the same property) we can assume $\psi\left(\left\{y_{n}\right\}\right)+\varepsilon \geq \rho\left(y_{n}-y_{m}\right)$ for every $n, m$. Define the following function from $[\mathbb{N}]^{2}$ into $\{1,2\}: f(n, m)=1$ if $\rho\left(y_{n}-y_{m}\right)>\psi\left(\left\{y_{n}\right\}\right)-\varepsilon$ and $f(n, m)=2$ if $\rho\left(y_{n}-y_{m}\right) \leq \psi\left(\left\{y_{n}\right\}\right)-\varepsilon$. By Ramsey's Lemma, there exists a subsequence $\left\{z_{n}\right\}$ of $\left\{y_{n}\right\}$ satisfying either $\rho\left(z_{n}-z_{m}\right)>\psi\left(\left\{y_{n}\right\}\right)-\varepsilon$ for every $n, m ; n \neq m$ or $\rho\left(z_{n}-z_{m}\right) \leq \psi\left(\left\{y_{n}\right\}\right)-\varepsilon$ for every $n, m$. Since the second possibility is a contradiction according to the property satisfied by $\left\{y_{n}\right\}$, we deduce that the first possibility always holds and we have

$$
\psi\left(\left\{y_{n}\right\}\right)-\varepsilon \leq \rho\left(z_{n}-z_{m}\right)<\psi\left(\left\{y_{n}\right\}\right)+\varepsilon
$$

for every $n, m ; n \neq m$. Choosing $\varepsilon=1 / n$, we can conclude the proof by a diagonal argument. 
Theorem 5.3. Let $\rho$ be a convex modular satisfying the $\Delta_{2}$-type condition. Assume that $\mathcal{X}_{\rho}$ is a $\phi-U N C$. Let $C$ be a nonempty closed bounded convex subset of the space $\mathcal{X}_{\rho}$ and let $\left\{x_{n}\right\}$ be a sequence in $C$ which is regular relative to $C$. Then there exists $\lambda<1$ depending on $r_{\rho}\left(C,\left\{x_{n}\right\}\right)$ such that

$$
r_{\rho}\left(C, A_{\rho}\left(C,\left\{x_{n}\right\}\right)\right) \leq \lambda r_{\rho}\left(C,\left\{x_{n}\right\}\right) .
$$

Proof. We are going to prove the result for $\phi=\alpha$. Denote $r=r_{\rho}\left(C,\left\{x_{n}\right\}\right)$ and $A=A_{\rho}\left(C,\left\{x_{n}\right\}\right)$. According to Theorem 5.2, we can find a subsequence $\left\{y_{n}\right\}$ of $\left\{x_{n}\right\}$ such that the limit $\lim _{n \neq m} \rho\left(y_{n}-y_{m}\right)$ exists. Take $z \in \cap_{n=1}^{\infty} \overline{c o}\left\{y_{k}\right.$ : $k \geq n\}$ which is a nonempty set due to property $(\mathrm{R})$. Since $\left\{x_{n}\right\}$ is regular relative to $C$, we have $r=r_{\rho}\left(C,\left\{y_{n}\right\}\right)$ and from Lemma 5.1, we obtain

$$
r \leq \limsup _{n} \rho\left(y_{n}-z\right) \leq \liminf _{m} \limsup _{n} \rho\left(y_{n}-y_{m}\right)=\lim _{n \neq m} \rho\left(y_{n}-y_{m}\right) .
$$

Hence, $\alpha\left(\left\{y_{n}\right\}\right) \geq r$. Thus, $\alpha\left(\overline{c o}\left\{y_{k}: k \geq n\right\}\right) \geq r$, for every $n \in \mathbb{N}$.

Assume $x$ lies in $A$. Consider $\lambda\left(r, \frac{1}{2}\right)<1$ and $\eta\left(r, \frac{1}{2}\right)>0$ given by Lemma 4.8. Since $r=\lim \sup \rho\left(y_{n}-x\right)$, for every $0<\varepsilon<\eta\left(r, \frac{1}{2}\right)$ there exists $n_{0} \in \mathbb{N}$ such that $\rho\left(y_{n}-x\right)<r+\varepsilon$ for all positive integer $n$ greater than or equal to $n_{0}$. Hence, the sequence $\left\{y_{n}\right\}_{n \geq n_{0}}$ is contained in the closed ball $B_{\rho}(x, r+\varepsilon)$ and $\alpha\left(\overline{c o}\left\{y_{k}: k \geq n\right\}\right) \geq(r+\bar{\varepsilon}) \frac{r}{r+\varepsilon}$. Therefore, for all $n \geq n_{0}$

$$
\begin{aligned}
d_{\rho}\left(x, \overline{c o}\left\{y_{k}: k \geq n\right\}\right) & \leq\left(1-\Delta_{\alpha}\left(r+\varepsilon, \frac{r}{r+\varepsilon}\right)\right)(r+\varepsilon) \\
& \leq\left(1-\Delta_{\alpha}\left(r+\varepsilon, \frac{1}{2}\right)\right)(r+\varepsilon)<\lambda\left(r, \frac{1}{2}\right)(r+\varepsilon) .
\end{aligned}
$$

In view of Theorem 4.9, we can find $\left.z_{n} \in \overline{c o}\left\{y_{k}: k \geq n\right\}\right)$ such that

$$
\rho\left(x-z_{n}\right)=d_{\rho}\left(x, \overline{c o}\left\{y_{k}: k \geq n\right\}\right) .
$$

Again by Theorem 4.10, $\cap_{n=n_{0}}^{\infty} \overline{c o}\left\{z_{k}: k \geq n\right\} \neq \emptyset$.

For each $w \in \cap_{n=n_{0}}^{\infty} \overline{c o}\left\{z_{k}: k \geq n\right\}$ and for all $n \geq n_{0}$, we have

$$
\rho(x-w) \leq \sup \left\{\rho(x-y): y \in \overline{c o}\left\{z_{k}: k \geq n\right\}\right\}=\sup \left\{\rho\left(x-z_{k}\right): k \geq n\right\} .
$$

Therefore,

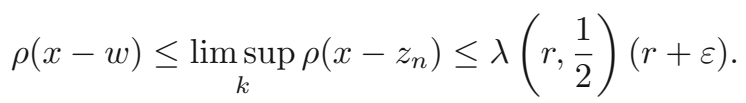

Since this inequality is true for every $\varepsilon>0$ and for every $x \in A$, we obtain the inequality in the statement.

Remark 5.4. Assume $0<d<r_{\rho}\left(C,\left\{x_{n}\right\}\right)$. If $r_{\rho}\left(C,\left\{x_{n}\right\}\right) \in\left(d, d+\eta\left(d, \frac{1}{2}\right)\right)$, we can follow the same argument as in Theorem 5.3 to obtain $\lambda(d)$ depending on $d$ such that

$$
r_{\rho}\left(C, A_{\rho}\left(C,\left\{x_{n}\right\}\right)\right) \leq \lambda(d) r_{\rho}\left(C,\left\{x_{n}\right\}\right)
$$




\section{Fixed point results}

Our fixed point results for multivalued $\rho$-nonexpansive mappings rely on the following proposition.

Proposition 6.1. Let $\rho$ be a convex modular satisfying the $\Delta_{2}$-type condition. Let $C$ be a nonempty closed bounded convex separable subset of the space $\mathcal{X}_{\rho}$ and $T: C \rightarrow K C_{\rho}(C)$ a $\rho$-nonexpansive mapping. Suppose $\left\{x_{n}\right\} \subset C$ is an approximate fixed point sequence for $T$, i.e., $\lim _{n} d_{\rho}\left(x_{n}, T x_{n}\right)=0$, and each subsequence of $\left\{x_{n}\right\}$ has a nonempty asymptotic center relative to $C$. Then there exists a subsequence $\left\{z_{n}\right\}$ of $\left\{x_{n}\right\}$ such that

$$
T x \cap A \neq \emptyset, \text { for all } x \in A:=A_{\rho}\left(C,\left(z_{n}\right)\right) .
$$

Proof. Since $C$ is separable, according to Lemma 2.13 there exists a subsequence $\left\{z_{n}\right\}$ of $\left\{x_{n}\right\}$ which is regular and asymptotically uniform with respect to $C$. Denote $r=r_{\rho}\left(C,\left\{z_{n}\right\}\right)$.

From the compactness of the set $T z_{n}$, we can find a sequence $\left\{u_{n}\right\}$ in $C$ such that $u_{n} \in T z_{n}$ and $\lim _{n \rightarrow \infty} \rho\left(z_{n}-u_{n}\right)=0$. Take any $x \in A$ and $v_{n} \in T x$ such that

$$
\rho\left(u_{n}-v_{n}\right)=d_{\rho}\left(u_{n}, T x\right) \leq H_{\rho}\left(T z_{n}, T x\right) \leq \rho\left(z_{n}-x\right) .
$$

Because of the $\rho$-compactness of $T x$, we can assume, by passing through a subsequence, that $\left\{v_{n}\right\}$ converges to a point $v \in T x$. From Lemma 2.9, we obtain

$$
\limsup _{n \rightarrow \infty} \rho\left(z_{n}-v\right)=\limsup _{n \rightarrow \infty} \rho\left(u_{n}-v_{n}\right) \leq \limsup _{n \rightarrow \infty} \rho\left(z_{n}-x\right)=r .
$$

This shows that $v \in A$, and so $T x \cap A \neq \emptyset$.

Now we are ready to prove an analogous result to the Kirk-Massa's theorem [25] in modular spaces.

Theorem 6.2. Let $\rho$ be a convex modular satisfying the $\Delta_{2}$-type condition. Assume that $C$ is a nonempty closed bounded convex subset of the space $\mathcal{X}_{\rho}$ and $T: C \rightarrow K C_{\rho}(C)$ a $\rho$-nonexpansive mapping. Suppose that each sequence in $C$ has a nonempty and compact asymptotic center relative to $C$. Then $T$ has a fixed point.

Proof. Since the $\Delta_{2}$-type condition is satisfied and $T$ is a continuous compact valued self-mapping, we can construct a closed convex subset of $C$ which is separable and invariant under $T$ (see [26]). Thus, we can suppose that $C$ is separable.

For a fixed element $x_{0} \in C$ and for each $n \geq 1$, define the mapping

$$
T_{n} x:=\frac{1}{n} x_{0}+\left(1-\frac{1}{n}\right) T x, \quad x \in C .
$$

We have that $T_{n}$ is a multivalued $\rho$-contraction and it has a fixed point $x_{n} \in C$ by Theorem 2.14. It is easily seen that $\lim _{n} d_{\rho}\left(x_{n}, T x_{n}\right)=0$. Without 
loss of generality, we may assume that $\left\{x_{n}\right\}$ is regular and asymptotically uniform with respect to $C$.

According to the previous proposition, we can also assume that

$$
T x \cap A \neq \emptyset, \quad \forall x \in A:=A_{\rho}\left(C,\left\{x_{n}\right\}\right) .
$$

Now we define the mapping $\tilde{T}: A \rightarrow K C_{\rho}(A)$ by $\tilde{T}(x)=T x \cap A$. Since $T$ is continuous, from Proposition 2.45 in [19], we know that the mapping $\tilde{T}$ is upper semicontinuous. Since $T x \cap A$ is a compact convex set we can apply the Kakutani-Bohnenblust-Karlin Theorem (see [16]) to obtain a fixed point for $\tilde{T}$ and so for $T$.

It should be pointed out that a modular $\rho$ satisfying (UUC1) has the property $(R)$ (see [1] and [21, Theorem 4.2]). If, in addition, the modular $\rho$ is uniformly continuous on bounded sets, it is easy to check that the asymptotic center of a sequence is nonempty and singleton. In view of this result, we can deduce the following corollary from Theorem 6.2.

Corollary 6.3. Let $\rho$ be a (UUC1) convex modular satisfying the $\Delta_{2}$-type condition. Assume that $C$ is a nonempty closed bounded convex subset of the space $\mathcal{X}_{\rho}$ and $T: C \rightarrow K C_{\rho}(C)$ a $\rho$-nonexpansive mapping. Then $T$ has a fixed point.

Definition 6.4. Let $\rho$ be a convex modular. A multivalued mapping $T: C \rightarrow$ $2^{\mathcal{X}_{\rho}}$ is called $\rho$ - $\phi$-contractive where $\phi$ is a measure of noncompactness if there exists a constant $k \in[0,1)$ such that for each $\rho$-bounded subset $B$ of $C$, we have

$$
\phi(T(B)) \leq k \phi(B)
$$

The following theorem states a relationship between $\rho$-contractive and $\rho$ $\chi_{C}-$ contractive mappings. In the framework of a Banach space, an analogous result was proved in [13].

Theorem 6.5. Let $\rho$ be a convex modular satisfying the $\Delta_{2}$-type condition and $C$ a nonempty closed subset of $\mathcal{X}_{\rho}$. Assume that $T: C \rightarrow K_{\rho}(C)$ is $\rho$ contractive with constant $k \in[0,1)$. Then $T$ is $\rho$ - $\chi_{C}$-contractive for the same constant $k$.

Proof. Let $A$ a bounded subset of $C, \varepsilon>0$ and $\mu>1$. Since $\mu>1$ we can take $\alpha \in(0,1)$ such that $\alpha \omega\left(\frac{1}{\alpha}\right) \leq \mu$. Now, we choose a number $\varepsilon^{\prime}>0$ such that

$$
\varepsilon^{\prime}<\min \left\{1, \frac{1}{\alpha \omega\left(\frac{1}{\alpha}\right)} \varepsilon, \frac{1}{(1-\alpha) \omega\left(\frac{1}{1-\alpha}\right)} \varepsilon\right\} .
$$

By definition of $\chi_{C}(A)$ there exists $a_{1}, \ldots a_{N} \in C$ such that

$$
A \subset \cup_{i=1}^{N} B_{\rho}\left(a_{i}, \chi_{C}(A)+\varepsilon^{\prime}\right) .
$$

On the other hand, since $T$ is compact valued, there exists $y_{1}, \ldots y_{n} \in C$ such that $\cup_{i=1}^{N} T a_{i} \subset \cup_{j=1}^{n} B\left(y_{j}, \varepsilon^{\prime}\right) \subset \cup_{j=1}^{n} B_{\rho}\left(y_{j}, \varepsilon^{\prime}\right)$. 
Let $x \in A$ and $i \in\{1, \ldots, N\}$ be such that $x \in B_{\rho}\left(a_{i}, \chi_{C}(A)+\varepsilon^{\prime}\right)$. Taking $y \in T x$, from the compactness of $T a_{i}$, we can find $z_{i} \in T a_{i}$ such that

$$
\rho\left(y-z_{i}\right)=d_{\rho}\left(y, T a_{i}\right) \leq H_{\rho}\left(T x, T a_{i}\right) \leq k \rho\left(x-a_{i}\right) \leq k\left(\chi_{C}(A)+\varepsilon^{\prime}\right) .
$$

Since $z_{i} \in T a_{i}$, we have $\rho\left(z_{i}-y_{j}\right) \leq \varepsilon^{\prime}$ for some $j \in\{1, \ldots, n\}$. Property (iii) of the modular and the definition of the growth function $\omega$ give

$$
\begin{gathered}
\rho\left(y-y_{j}\right) \leq \alpha \omega\left(\frac{1}{\alpha}\right) \rho\left(y-z_{i}\right)+(1-\alpha) \omega\left(\frac{1}{1-\alpha}\right) \rho\left(z_{i}-y_{j}\right) \leq \\
\alpha \omega\left(\frac{1}{\alpha}\right) k\left(\chi_{C}(A)+\varepsilon^{\prime}\right)+(1-\alpha) \omega\left(\frac{1}{1-\alpha}\right) \varepsilon^{\prime} .
\end{gathered}
$$

Thus $\rho\left(y-y_{j}\right) \leq \mu k \chi_{C}(A)+2 \varepsilon$. Hence, $\chi_{C}(T(A)) \leq \mu \chi_{C}(A)+2 \varepsilon$. Since $\varepsilon$ is arbitrary we have $\chi_{C}(T(A)) \leq \mu \chi_{C}(A)$ for all $\mu>1$. Thus, $\chi_{C}(T(A)) \leq k \chi_{C}(A)$, obtaining the desired result.

Theorem 6.6. Let $\rho$ be a convex modular satisfying the $\Delta_{2}$-type condition and $C$ a nonempty closed convex subset of $\mathcal{X}_{\rho}$. Assume that $T: C \rightarrow K C_{\rho}(C)$ is a $\rho$-contractive mapping with constant $k \in[0,1)$. Suppose that $A$ is a closed bounded convex subset of $C$ such that $T x \cap A \neq \emptyset$ for every $x \in A$. Then $T$ has a fixed point in $A$.

Proof. According to Theorem 6.5, the mapping $T$ is $\rho$ - $\chi_{C}$-contractive. Denote $A_{1}=A$ and assume that we have defined a finite decreasing sequence of closed convex sets $A_{n} \subset A_{n-1} \subset \ldots \subset A_{1}$ such that $T x \cap A_{j} \neq \emptyset$ for every $x \in A_{j}$ and $\chi_{C}\left(A_{j}\right) \leq k \chi_{C}\left(A_{j-1}\right)$ for all $j=1, \ldots, n$. Define $A_{n+1}=\left[\overline{c o} T\left(A_{n}\right)\right] \cap A_{n}$. Then, $A_{n+1}$ is a closed convex subset of $A_{n}$. Furthermore, for every $x \in A_{n+1}$, we have $T x \cap A_{n} \neq \emptyset$. Since $T x \subset T\left(A_{n}\right)$, we obtain that $T x \cap A_{n+1}$ is nonempty. On the other hand,

$$
\begin{aligned}
\chi_{C}\left(A_{n+1}\right) & =\chi_{C}\left(\overline{c o}\left(T\left(A_{n}\right)\right) \cap A_{n}\right) \leq \chi_{C}\left(\overline{c o}\left(T\left(A_{n}\right)\right)\right) \\
& =\chi_{C}\left(\operatorname{co}\left(T\left(A_{n}\right)\right)=\chi_{C}\left(T\left(A_{n}\right)\right)\right. \\
& \leq k \chi_{C}\left(A_{n}\right),
\end{aligned}
$$

Since $k<1, \lim _{n} \chi_{C}\left(A_{n}\right)=0$ and $A_{\infty}=: \cap_{n=1}^{\infty} A_{n}$ is a nonempty compact convex subset of $A$.

Let $x \in A_{\infty}$ and take $a_{n} \in T x \cap A_{n}$ which is nonempty. Since $\chi_{C}\left(\left\{a_{n}\right\}\right)=$ $\chi_{C}\left(\left\{a_{k}: k \geq n\right\}\right) \leq \chi_{C}\left(A_{n}\right)$, the sequence $\left\{a_{n}\right\}$ has some cluster point $a \in A_{\infty}$. On the other hand, the sequence $\left\{a_{n}\right\}$ lies in $T x$ which is a compact set implying that $a$ belongs to $T x$. Thus, $T x \cap A_{\infty} \neq \emptyset$. Since $A_{\infty}$ is compact convex we apply Kakutani-Bohnenblust-Karlin Theorem to obtain that $T$ has a fixed point in $A_{\infty} \subset A$.

We state now the main fixed point result in this paper. 
Theorem 6.7. Let $\rho$ be a convex modular satisfying the $\Delta_{2}$-type condition. Assume that $\mathcal{X}_{\rho}$ is $\alpha$-UNC and for some $\varepsilon_{0} \in(0,1)$, there exists $\gamma>0$ such that $\lim _{r \rightarrow 0} \Delta_{\alpha}\left(r, \varepsilon_{0}\right) \geq \gamma$. Let $C$ be a nonempty closed bounded convex subset of the space $\mathcal{X}_{\rho}$ and $T: C \rightarrow K C_{\rho}(C)$ a $\rho$-nonexpansive mapping. Then $T$ has a fixed point.

Proof. As in Theorem 6.2 we can suppose that $C$ is separable. First, we claim that for any closed convex subset $A$ of $C$ such that $T x \cap A \neq \emptyset$ for every $x \in A$, there exists an approximated fixed point sequence of $T$ in $A$, i.e. there exists $\left\{x_{n}\right\} \subset A$ such that $d_{\rho}\left(x_{n}, T x_{n}\right) \rightarrow 0$. Indeed, let $x_{0} \in A$ be fixed and, for each $n \geq 1$, define

$$
T_{n} x:=\frac{1}{n} x_{0}+\left(1-\frac{1}{n}\right) T x, \quad x \in C .
$$

Since $T_{n}$ is $(1-1 / n)$-contractive and $T_{n} x \cap A \neq \emptyset, T_{n}$ has a fixed point $x_{n} \in A$ from Theorem 6.6. It is easy to deduce that $\lim _{n} d_{\rho}\left(x_{n}, T x_{n}\right)=0$. Without loss of generality, we may assume that $\left\{x_{n}\right\}$ is regular and asymptotically uniform with respect to $C$. On the other hand, we can apply Theorem 5.3 to obtain

$$
r_{\rho}\left(C, A_{\rho}\left(C,\left\{x_{n}\right\}\right)\right) \leq \lambda(r) r,
$$

where $r=r_{\rho}\left(C,\left\{x_{n}\right\}\right.$ and $\lambda(r)<1$. Now, we follow a similar proof to that in [12] (Theorem 4.1). In the first step we proceed as in Theorem 6.2 to obtain a set $A_{1}$ such that $T x \cap A_{1} \neq \emptyset$. According to the above claim, we may assume that sets $A_{2}, \ldots, A_{m}$ and approximated fixed point sequences $\left\{x_{n}^{k}\right\} \subset A_{k}$ are constructed where $A_{k}=A_{\rho}\left(C,\left\{x_{n}^{k-1}\right\}\right), r_{k}=r_{\rho}\left(C,\left\{x_{n}^{k-1}\right\}, \lambda\left(r_{k}\right)<1\right.$ and

$$
r_{\rho}\left(C, A_{k}\right) \leq \lambda\left(r_{k}\right) r_{k} \leq \lambda\left(r_{k}\right) r_{\rho}\left(C, A_{k-1}\right)
$$

for $k=2, \ldots, m$. Defining $A_{m+1}=A_{\rho}\left(C,\left\{x_{n}^{m}\right\}\right)$ and choosing a suitable approximated fixed point sequence $\left\{x_{n}^{m+1}\right\}$ in $A_{m+1}$, we obtain

$$
r_{\rho}\left(C, A_{m+1}\right) \leq \lambda\left(r_{m+1}\right) r_{m+1} \leq \lambda\left(r_{m+1}\right) r_{\rho}\left(C, A_{m}\right) \leq \lambda\left(r_{m+1}\right) \lambda\left(r_{m}\right) r_{m}
$$

and we can continue the induction process.

Hence, $\left\{r_{m}\right\}$ is a non-decreasing sequence. Suppose that $d:=\lim _{m} r_{m}>$ 0 . Choose $\eta\left(d, \frac{1}{2}\right)>0$ given by Lemma 4.8 . Without loss of generality, we can suppose that $r_{m} \in\left(d, d+\eta\left(d, \frac{1}{2}\right)\right)$ for every $m \geq 1$. By Remark 5.4 , we have that $r_{m+1} \leq r_{\rho}\left(C, A_{m}\right) \leq \lambda(d) r_{m}$, where $\lambda(d)<1$. Taking limit as $m \rightarrow \infty$ we have $d \leq \lambda(d) d$. This contradicts the fact that $d>0$. Thus, $\lim _{m} r_{m}=0$. Since $\lim _{r \rightarrow 0} \Delta_{\alpha}\left(r, \varepsilon_{0}\right) \geq \gamma$ and $\left\{r_{m}\right\}$ is a non-decreasing sequence, we proceed as in Theorem 5.3 to get

$$
r_{\rho}\left(C, A_{m}\right) \leq \lambda r_{m},
$$

for $m$ sufficient large, where $\lambda=1-\gamma$. This implies that

$$
r_{\rho}\left(C, A_{m}\right) \leq \lambda^{m-1} r_{\rho}\left(C, A_{1}\right)
$$


Choose $x_{m} \in A_{m}$. We shall prove that $\left\{x_{m}\right\}_{m}$ is a norm-Cauchy sequence. For $m$ sufficiently large we have for any positive integer $n$

$$
\begin{aligned}
\rho\left(x_{m}-x_{m+1}\right) & \leq \frac{\omega(2)}{2}\left(\rho\left(x_{m}-x_{n}^{m}\right)+\rho\left(x_{n}^{m}-x_{m+1}\right)\right) \\
& \leq \frac{\omega(2)}{2}\left(\operatorname{diam}_{\rho} A_{m}+\rho\left(x_{n}^{m}-x_{m+1}\right)\right) .
\end{aligned}
$$

Taking upper limit as $n \rightarrow \infty$,

$$
\begin{aligned}
\rho\left(x_{m}-x_{m+1}\right) & \leq \frac{\omega(2)}{2}\left(\operatorname{diam}_{\rho} A_{m}+\limsup _{n} \rho\left(x_{n}^{m}-x_{m+1}\right)\right) \\
& =\frac{\omega(2)}{2}\left(\operatorname{diam}_{\rho} A_{m}+r_{\rho}\left(C,\left\{x_{n}^{m}\right\}\right)\right) \\
& \leq \frac{\omega(2)}{2}\left(\operatorname{diam}_{\rho} A_{m}+r_{\rho}\left(C, A_{m}\right)\right) \\
& \leq \frac{\omega(2)}{2}\left(\omega(2) r_{\rho}\left(C, A_{m}\right)+r_{\rho}\left(C, A_{m}\right)\right. \\
& =\frac{\omega(2)}{2}(\omega(2)+1) r_{\rho}\left(C, A_{m}\right) \\
& \leq \frac{\omega(2)}{2}(\omega(2)+1) \lambda^{m-1} r_{\rho}\left(C, A_{1}\right)
\end{aligned}
$$

Let $M=\frac{\omega(2)}{2}(\omega(2)+1) r_{\rho}\left(C, A_{1}\right)$, then

$$
\frac{1}{\lambda^{m-1} M} \leq \frac{1}{\rho\left(x_{m}-x_{m+1}\right)} .
$$

Property (4) in Lemma 2.9 implies

$$
\left(\omega^{-1}\left(\frac{1}{\lambda}\right)\right)^{m-1} \omega^{-1}\left(\frac{1}{M}\right) \leq \omega^{-1}\left(\frac{1}{\rho\left(x_{m}-x_{m+1}\right)}\right),
$$

and from property (6) of the same lemma, we obtain

$$
\left\|x_{m+1}-x_{m}\right\|_{\rho} \leq \frac{1}{\omega^{-1}\left(\frac{1}{\rho\left(x_{m}-x_{m+1}\right)}\right)} \leq\left(\frac{1}{\omega^{-1}\left(\frac{1}{\lambda}\right)}\right)^{m-1} \frac{1}{\omega^{-1}\left(\frac{1}{M}\right)} .
$$

Since $\omega^{-1}$ is strictly increasing, we have $\frac{1}{\omega^{-1}\left(\frac{1}{\lambda}\right)}<1$. This implies that $\left\{x_{m}\right\}$ is a norm-Cauchy sequence and so norm-convergent to a point $x \in C$. Thus, the sequence $\left\{x_{m}\right\}$ is $\rho$-convergent to $x$.

Let us see that $x$ is a fixed point of $T$. For each $m \geq 1$, it is not difficult to check that

$$
\begin{aligned}
d_{\rho}\left(x_{m}, T x_{m}\right) & \leq \omega(3)\left(\rho\left(x_{m}-x_{n}^{m}\right)+d_{\rho}\left(x_{n}^{m}, T x_{n}^{m}\right)+H_{\rho}\left(T x_{n}^{m}, T x_{m}\right)\right) \\
& \leq \omega(3)\left(2 \rho\left(x_{m}-x_{n}^{m}\right)+d_{\rho}\left(x_{n}^{m}, T x_{n}^{m}\right)\right) .
\end{aligned}
$$

Taking upper limit as $n \rightarrow \infty$

$$
d_{\rho}\left(x_{m}, T x_{m}\right) \leq 2 \omega(3) \limsup _{n} \rho\left(x_{m}-x_{n}^{m}\right) \leq 2 \omega(3) \lambda^{m-1} r_{\rho}\left(C, A_{1}\right) .
$$


Taking limit in $m$ in both sides we obtain $\lim _{m} d_{\rho}\left(x_{m}, T x_{m}\right)=0$. In a similar way we can prove that $d_{\rho}(x, T x)=0$, i.e. $x \in T x$. Indeed, we have

$$
\begin{aligned}
d_{\rho}(x, T x) & \leq \omega(3)\left(\rho\left(x-x_{m}\right)+d_{\rho}\left(x_{m}, T x_{m}\right)+H_{\rho}\left(T x_{m}, T x\right)\right) \\
& \leq \omega(3)\left(2 \rho\left(x_{m}-x\right)+d_{\rho}\left(x_{m}, T x_{m}\right)\right)
\end{aligned}
$$

and letting $m \rightarrow \infty$ we get the desired result.

The above theorem extends the Kirk-Massa theorem in modular spaces, namely Theorem 6.2, in the sense that we do not need the compactness of asymptotic center of a sequence. We illustrate this fact by means of the following example.

Example 6.8. A uniformly $\rho$-noncompact convex space with non-compact asymptotic centers

Let $\left\{p_{n}\right\}$ be as in Example 4.7 and define $\sigma: \ell^{p_{n}} \rightarrow[0, \infty)$ by

$$
\sigma(x)=\sup _{n}\left\{|x(n)|^{p_{n}}+\frac{1}{2} \sum_{k=n+1}^{\infty}|x(k)|^{p_{k}}\right\} .
$$

It is clear that $\sigma$ is a convex modular and $\left(\ell^{p_{n}}, \sigma\right)$ satisfies the $\Delta_{2}$-type condition. Furthermore,

$$
\frac{1}{2} \rho(x) \leq \sigma(x) \leq \rho(x)
$$

which implies that the Luxemburg norms $\|\cdot\|_{\sigma}$ and $\|\cdot\|_{\rho}$ are equivalent. (Nominally,

$$
\|x\|_{\sigma} \leq\|x\|_{\rho} \leq 2^{1 / P}\|x\|_{\sigma},
$$

where $\left.P=\limsup _{n} p_{n}\right)$. Thus, $\left(\ell^{p_{n}},\|\cdot\|_{\sigma}\right)$ is a reflexive Banach space and, as in Example 4.7, any weakly null sequence converges to zero coordinatewise.

Claim. Assume that $\operatorname{supp} x \leq N<\operatorname{supp} y$. Then $\sigma(x+y) \leq \sigma(x)+\sigma(y)$. Furthermore, $\sigma(x)+(1 / 2) \sigma(y) \leq \sigma(x+y)$ if $\sigma(x)>\sigma(y)$.

To prove the claim, note that $\max \{\sigma(x), \sigma(y)\} \leq \sigma(x+y) \leq \sigma(x)+\sigma(y)$. Assume that there exists $k>N$ such that

$$
\sigma(x+y)=|y(k)|+\frac{1}{2} \sum_{n=k+1}^{\infty}|y(n)| .
$$

In this case, we have $\sigma(x) \leq \sigma(x+y)=\sigma(y)$. Thus, the condition $\sigma(x)>\sigma(y)$ implies that there exists $k \leq N$ such that

$$
\begin{aligned}
\sigma(x+y) & =|x(k)|+\frac{1}{2} \sum_{i=k+1}^{N}|x(i)|+\frac{1}{2} \sum_{i=N+1}^{\infty}|y(i)| \\
& =\sigma(x)+\frac{1}{2} \rho(y) \geq \sigma(x)+\frac{1}{2} \sigma(y) .
\end{aligned}
$$

Assume that $A$ is a convex subset of the closed ball $B_{\sigma}(0, r)$ such that $\chi(A)>\varepsilon r$. As in Example 4.7, we find sequences $\left\{x_{n}\right\}$ in $A$ and $\left\{u_{n}\right\},\left\{v_{n}\right\}$ such that $\operatorname{sep}_{\sigma}\left(x_{n}\right) \geq \varepsilon r,\left\{x_{n}\right\}$ is weakly convergent, say to $x$, and $\lim _{n} \sigma\left(x_{n}-\right.$ 
$x)=: \operatorname{lr}$ does exist; $\lim _{n} \sigma\left(x-u_{n}\right)=0, \lim _{n} \sigma\left(x_{n}-x-v_{n}\right)=0, \operatorname{supp} u_{n}<$ $\operatorname{supp} v_{n}$ and $\operatorname{supp} v_{n}<\operatorname{supp} v_{n+1}$ for every $n \in \mathbb{N}$. For an arbitrary $\eta>0$, we can choose $n, m$ large enough such that (1.1)-(1.4) in Example 4.7 are satisfied (replacing $\rho$ by $\sigma$ ). The same argument as in Example 4.7 proves that $\varepsilon r-\eta<\sigma\left(v_{n}-v_{m}\right) \leq 2 l r+2 \eta$.

We split the remaining part of the proof in two cases: (1) Assume $\sigma(x) \leq$ $l r$. In this case, there exists $n \in \mathbb{N}$ such that $\sigma\left(u_{n}\right) \leq \sigma\left(v_{n}\right)+\eta$ and we have

$$
r+\eta \geq \sigma\left(x_{n}\right)+\eta \geq \sigma\left(v_{n}\right)+\sigma\left(u_{n}\right)+\eta \geq 2 \sigma\left(u_{n}\right)+2 \eta
$$

which implies $\sigma(x) \leq r / 2$. (2) Assume $\sigma(x)>l r$. There exists $n \in \mathbb{N}$ such that $\sigma\left(u_{n}\right)>\sigma\left(v_{n}\right)$ and we have

$$
\begin{aligned}
r \geq \sigma\left(x_{n}\right) & \geq \sigma\left(v_{n}+u_{n}\right)-\eta \geq \sigma\left(u_{n}\right)+\frac{1}{2} \sigma\left(v_{n}\right)-\eta \geq l r+\sigma(x)-3 \eta \\
& \geq \frac{r \varepsilon}{4}+\sigma(x)-6 \eta
\end{aligned}
$$

which implies $\Delta_{\chi}(\varepsilon, r) \geq \varepsilon / 4$.

Finally, considering the sequence $\left\{e_{n}\right\}$, it is clear that $\liminf _{n} \sigma\left(e_{n}-\right.$ $x) \geq 1$ for every $x \in \ell^{p_{n}}$. Thus, $r_{\rho}\left(B_{\sigma}(0,1),\left\{e_{n}\right\}\right)=1$. Note that $\sigma\left(e_{n}-\right.$ $\left.(1 / 2) e_{k}\right)=1$ for every $k<n$ which implies that the sequence $\left\{e_{n} / 2\right\}$ lies in the asymptotic center of $\left\{e_{n}\right\}$ with respect to the unit ball.

Funding Open Access funding provided thanks to the CRUE-CSIC agreement with Springer Nature.

Open Access. This article is licensed under a Creative Commons Attribution 4.0 International License, which permits use, sharing, adaptation, distribution and reproduction in any medium or format, as long as you give appropriate credit to the original author(s) and the source, provide a link to the Creative Commons licence, and indicate if changes were made. The images or other third party material in this article are included in the article's Creative Commons licence, unless indicated otherwise in a credit line to the material. If material is not included in the article's Creative Commons licence and your intended use is not permitted by statutory regulation or exceeds the permitted use, you will need to obtain permission directly from the copyright holder. To view a copy of this licence, visit http:// creativecommons.org/licenses/by/4.0/.

Publisher's Note Springer Nature remains neutral with regard to jurisdictional claims in published maps and institutional affiliations.

\section{References}

[1] Abdou, A.A.N., Khamsi, M.A.: Fixed point theorems in modular vector spaces. J. Nonlinear Sci. Appl. 10(8), 4046-4057 (2017)

[2] Ayerbe, J.M., Domínguez Benavides, T., López Acedo, G.: Measures of Noncompactness in Metric Fixed Point Theory. Birkhäuser (1997)

[3] Bachar, M., Bounkhel, M., Khamsi, M.A.: Uniform Convexity in lp(.). J. Nonlinear Sci. Appl. 10, 5292-5299 (2017) 
[4] Bachar, M., Mendez, O., Bounkhel, M.: Modular uniform convexity of Lebesgue spaces of variable integrability. Symmetry 10(12), 708 (2018). https://doi.org/ 10.3390/sym10120708

[5] Browder, F.E.: Fixed point theorems for noncompact mappings in Hilbert spaces. Proc. Nat. Acad. Sci. USA 43, 1272-1276 (1965)

[6] Browder, F.E.: Nonexpansive nonlinear opemtors in a Banach space. Proc. Nat. Acad. Sci. USA 54, 1041-1044 (1965)

[7] Dhompongsa, S., Domínguez Benavides, T., Kaewcharoen, A., Panyanak, B.: Fixed point theorems for multivalued mappings in modular function spaces. Sci. Math. Jpn. 63(2), 161-169 (2006)

[8] Domínguez Benavides, T.: Geometric properties of Banach spaces and metric fixed point theory. Extracta Math. 17, 331-349 (2002)

[9] Domínguez Benavides, T., Gavira, B.: Does Kirk's theorem holds for multivalued nonexpansive mappings? Fixed Point Theory Appl. 2010, 546761 (2010). https://doi.org/10.1155/2010/546761

[10] Domínguez Benavides, T., Japón, M.: Fixed point properties and reflexivity in variable Lebesgue spaces. J. Funct. Anal. 280(6), 108896 (2021). https://doi. org/10.1016/j.jfa.2020.108896

[11] Domínguez Benavides, T., Khamsi, M.A., Samadi, S.: Asymptotically regular mappings in modular function spaces. Sci. Math. Jpn. 53(2), 295-304 (2001)

[12] Domínguez Benavides, T., Lorenzo, P.: Fixed point theorems for multivalued nonexpansive mappings without uniform convexity. Abs. Appl. Anal. 6(2003), 375-386 (2003)

[13] Domínguez Benavides, T., Lorenzo, P.: Asymptotic centers and fixed points for multivalued nonexpansive mappings. Ann. Univ. Mariae Curie-Sklodowska Sect. A 58, 37-45 (2004)

[14] Domínguez Benavides, T., Moshtaghioun, S.M., Sadeghi Hafshejani, A.: Fixed points for several classes of mappings in Variable Lebesgue Spaces. Optimization. https://doi.org/10.1080/02331934.2019.1711086

[15] Goebel, K.: On a fixed point theorem for multivalued nonexpansive mappings. Ann. Univ. Marie Curie-Sklodowska 29, 70-72 (1975)

[16] Goebel, K., Kirk, W.A.: Topics in Metric Fixed Point Theory. Cambridge University Press (1990)

[17] Goebel, K., Sekowski, T.: The modulus of noncompact convexity. Ann. Univ. Mariae Curie-Sklodowska Sect. A 38, 41-48 (1984)

[18] Göhde, D.: Zum Prinzip der kontraktiven Abbildung. Math. Nach. 30, 251-258 (1965)

[19] Hu, S., Papageorgiou, N.: Handbook of Multivalued Analysis, vol. 1. Kluwer Academic Publishers, Dordrecht (1997)

[20] Kamińska, A.: On unifrom convexity of Orlicz spaces. Nederl. Akad. Wetensch. Indag. Math. 44(1), 27-36 (1982)

[21] Khamsi, M.A., Kozlowski, W.M.: Fixed Point Theory in Modular Function Spaces. Birkäuser, Basel (2015)

[22] Khamsi, M.A., Kozlowski, W.M., Reich, S.: Fixed point theory in modular function spaces. Nonlinear Anal. 14(11), 935-953 (1990)

[23] Kozlowski, W.M.: Modular function spaces. Dekker, New York (1988) 
[24] Kirk, W.A.: A fixed point theorem for mappings which do not increase the distances. Am. Math. Mon. 72, 1004-1006 (1965)

[25] Kirk, W.A., Massa, S.: Remarks on asymptotic and Chebyshev centers. Houston J. Math. 16(3), 357-364 (1990)

[26] Kuczumov, T., Prus, S.: Asymptotic centers and fixed points of multivalued nonexpansive mappings. Houst. J. Math. 16, 465-468 (1990)

[27] Lim, T.C.: A fixed point theorem for multivalued nonexpansive mappings in a uniformly convex Banach space. Bull. Am. Math. Soc. 80, 1123-1126 (1974)

[28] Musielak, J., Orlicz, W.: On modular spaces. Studia Math. 18, 591-597 (1959)

[29] Nakano, H.: Modulared Semi-ordered Linear Spaces. Maruzen Co., Tokyo (1950)

[30] Nakano, H.: Topology of Linear Topological Spaces. Maruzen Co. Ltd, Tokyo (1951)

[31] Orlicz, W.: Über konjugierte Exponentenfolgen. Studia Math. 3, 200-212 (1931)

[32] Reich, S.: Some fixed point problems. Atti Accad. Naz. Lincei 57, 194-198 (1974)

[33] Reich, S.: Approximate selections, best approximations, fixed points, and invariant sets. J. Math. Anal. Appl. 62, 104-113 (1978)

[34] Rǔžička, M.: Electrorheological Fluids: Modeling and Mathematical Theory. Lecture Notes in Mathematics, vol. 1748. Springer, Berlin (2000)

T. Domínguez Benavides and P. Lorenzo Ramírez

Departamento de Análisis Matemático

Universidad de Sevilla

P. O. Box 1160Seville 41080

Spain

e-mail: tomasd@us.es;

ploren@us.es

Accepted: June 11, 2021. 